\title{
Diabète sucré et autoimmunité
}

La valeur de l'hypothèse autoimmune dans la genèse du diabète insulinodépendant juvénile se trouve confortée par différents apports récents : recherche directe d'anticorps et de cellules cytotoxiques, association avec des marqueurs sériques et membranaires, description de modèles animaux, résultats des thérapeutiques immunosuppressives.

\section{Roger Assan \\ Professeur à la Faculté de méde- cine Xavier-Bichat \\ Chef du service de diabétologie de l'hôpital Bichat}

\section{REFERENCES}

I. Carpenter CCJ, Solomon N, Silberberg SG, et al. Schmidt's syndrome: a review of the literature and a report of fifteen new cases including ten instances of coexisting diabetes mellitus. Medicine (Baltimore) 1964;43: 153 .

2. Irvinc WJ, Clarkc BF, Scarth L, Cullen DR, Duncan LJP. Thyroid and gastric autoimmunity in patients with diabetes mellitus. Lancet 1970; ii : 163-8.

\section{ADRESSE}

R. Assan : Hôpital Bichat, 18, rue Henri- e syndrome d'hyperglycémie chronique communément dénommé diabète sucré relève de causes diverses, correspondant à des maladies différentes. Déficit de l'insulino-sécrétion et résistance des tissus cibles à l'insuline sont les deux éléments physiopathologiques que l'on retrouve en proportions inégales dans tous ces syndromes (figure 1). Le diabète insulinodépendant dans sa forme la plus fréquente en milieu occidental est la conséquence d'une destruction des cellules sécrétant l'insuline (cellules bêta-langerhansiennes), où l'autoimmunité anti-îlot de Langerhans paraît jouer un rôle prépondérant. C'est à cet aspect nouveau de la physiopathologie, où les connaissances évoluent rapidement, qu'est consacrée la présente synthèse, à l'exclusion d'autres manifestations autoimmunes plus rares: autoanticorps anti-récepteurs de l'insuline, auto-anticorps anti-insuline endogène.

Le concept de diabète immunité anti-îlot s'est forgé à partir d'arguments cliniques, morphologiques, génétiques, et immunologiques. Il s'y ajoute depuis peu deux autres séries d'arguments: des modèles animaux de diabète spontané insulinoprive avec immunité anti-îlot, et l'obtention de rémission du diabète (et de l'insulite) grâce à un traitement immunosuppresseur, chez l'homme aussi bien que dans un modèle animal de diabète spontané.

\section{Argument clinique -}

Au diabète de type 1 (diabète insulinodépendant) peuvent s'associer diverses affections où l'autoimmunité intervient avec une fréquence supérieure à celle rencontrée dans une population non sélectionnée (tableau I). Certaines de ces associations morbides concernent des affections endocriniennes autoimmunes : thyroïdite de Hashimoto, maladie de Basedow, myxoedème, exophtalmie basedowienne sans hyperthyréose, insuffisance surrénale chronique par rétraction corticale, et peut être aussi l'aménorrhée précoce avec alopécie et l'insuffisance parathyroidienne. D'autres associations concernent des affections autoimmunes non endocriniennes : anémie pernicieuse de Biermer, myasthénie avec autoanticorps antirécepteur de l'acétylcholine, vitiligo, hépatite chronique active (tableau II).

$\mathrm{La}$ fréquence de ces associations morbides est diversement appréciée selon les critères cliniques ou biologiques retenus. Plus grande encore est la fréquence d'une association au diabète sucré de mani- 
festations infra-cliniques : la présence dans le sérum d'anticorps anti-thyroïde, anti-surrénale, antimuqueuse gastrique etc. Depuis longtemps relevées par les cliniciens [1] et les immunologistes [2], ces associations morbides tirent leurs valeurs de la coexistence de manifestations immunologiques depuis longtemps définies, et du diabète sucré insulinodépendant.

\section{Arguments anatomiques}

Dans le diabète sucré non insulinodépendant de la maturité (diabète de type 2), le pancréas dans son ensemble est normal. Les îlots de Langerhans ont une morphologie sensiblement normale qualitativement et quantitativement. Il en va tout différemment dans le diabète de type 1. Le pancréas dans son ensemble présente une réduction de poids et de taille. Cette réduction porte électivement sur les zones où les îlots sont riches en cellules bêtalangheransiennes (sécrétant l'insuline) et, au contraire, les zones où prédominent les îlots à cellules PP (sécrétant le polypeptide pancréatique) sont respectées. Les îlots de Langerhans lorsque le diabète a évolué pendant plusieurs années, sont à peine visibles, réduits à quelques cordons cellulaires perdus dans un stroma fibreux : on parle d'îlots "pseudo-atrophiques ». On y reconnait presque exclusivement des cellules A (sécrétant le glucagon), D (sécrétant le somatostatine) et PP (sécrétant le polypeptide pancréatique). Lorsque le pancréas est examiné peu de mois ou de semaines après l'apparition clinique du diabète, les îlots sont de taille variable, certains sont normaux ou même hypertrohiques, ils contiennent des cellules béta augmentées de volume, hydropiques, chargées de glycogène. Certains îlots surtout sont le siège d'une infiltration par des lymphocytes disloquant l'architecture normale de l'îlot. Cette insulite est caractéristique du diabète récemment diagnostiqué de l'adulte jeune. La fréquence de l'insulite varie selon les observations. W. Gepts l'a observée chez 16 des 23 diabétiques insulinodépendants dont le pancréas avait été examiné $m / s n^{\circ} 4$ juin-juillet 85
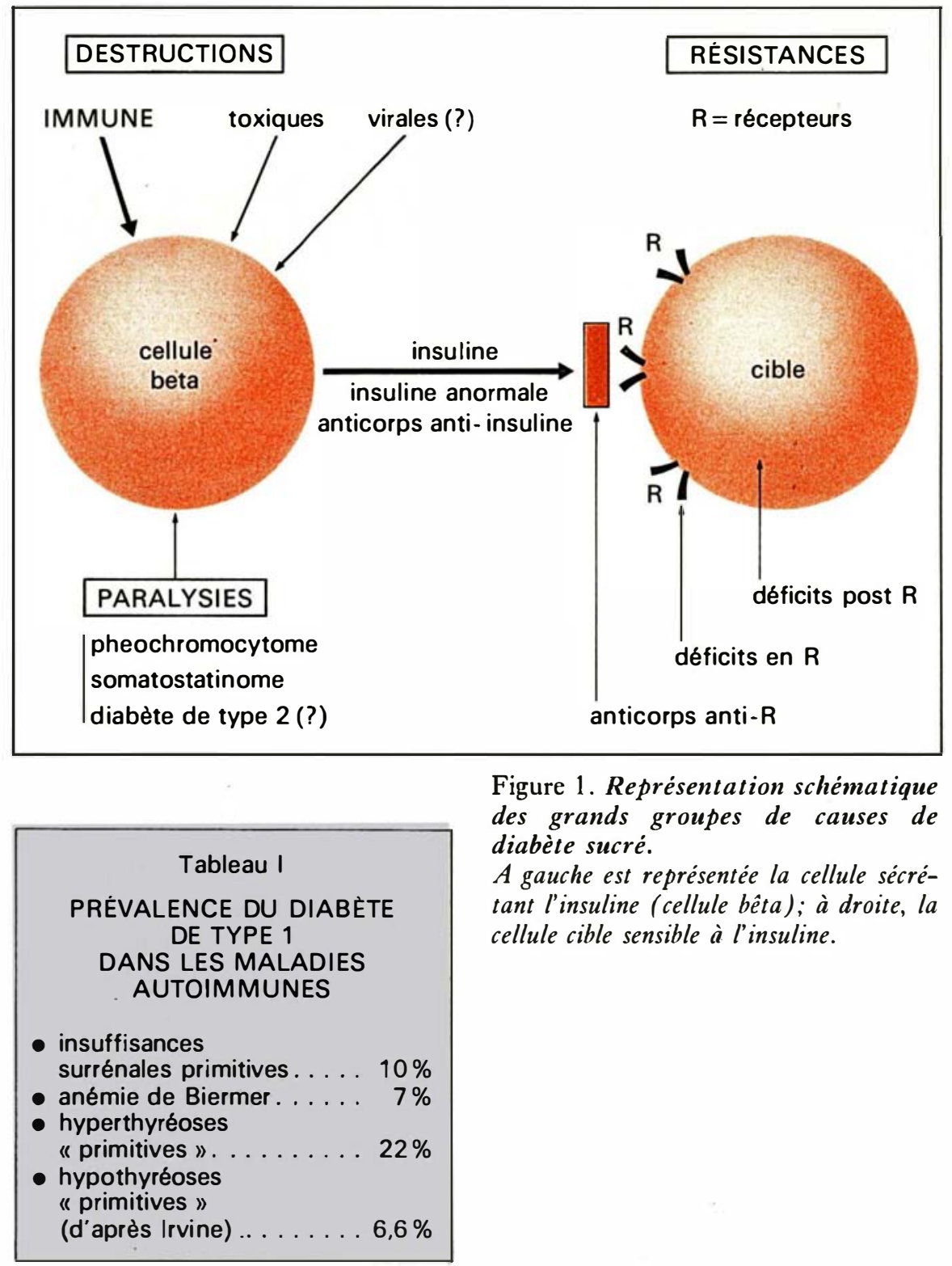

Figure 1. Représentation schématique des grands groupes de causes de diabète sucré.

A gauche est représentée la cellule sécrétant l'insuline (cellule bêta); à droite, la cellule cible sensible à l'insuline.

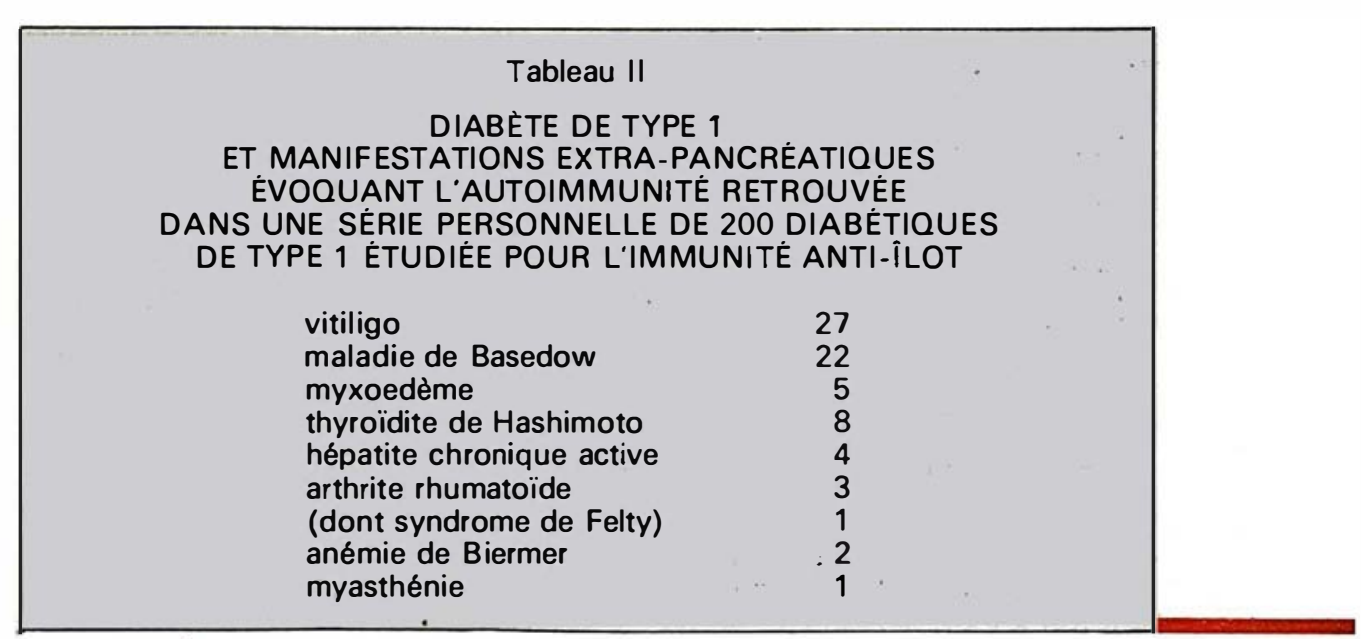




\section{REFERENCES}

3. Gepts W Pathology of the pancreas in juvenile diabetes. Diabetes 1965; 14:619.

4. Botazzo GF, Pujol-Borrell R, Hanfusa T, Feldmann M. Role of aberrant HLA-DR expression and antigen presentation in induction of endocrine autoimmunity. Lancet 1983 ; ii : 1 I 5-9.

5. Ballardini G, Bianchi FB, Doniach D, Mirakian R, Pisi E, Bottazzo GF. Aberrant expression of HLA-DR antigens on bileduct epithelium in primary biliary cirrhosis : relevance to pathogenesis. Lancet 1984 , i : 1009-1 3 .

6. Yoon JW, Austin M, Onodera T, Notkins AL. Virus induced diabetes mellitus : isolation of a virus from the pancreas of a child with diabetic ketoacidosis. N Engl 7 Med 1979; 300 : 1 $173-9$.

7. Gepts W. The pathology of the pancreas in human diabetes. In : Andreani D, Di Mario U, Federlin KF, Heding LG, eds.Immunology in diabetes. London: Kimpton Medical Publications, $1984: 21-34$

8. Onodera T, Ray UR, Melez KA, Suzuki $H$, Toniolo A, Notkins AL. Virus induced diabetes mellitus : autoimmunity and polyendocrine disease prevented by immuno-suppression. Nature 1982; $297: 66-8$.

9. Dean B, Pujol-Borrell R, Botazzo GF Determination of islet cell antibodies by immunofluorescence. Lancet 1982; ii : 1343-4.

10. Brogren $\mathrm{CH}$, Lernmark $\mathrm{A}$. Islet cell antibodies in diabetes. Clin Endocrinol Metab 1982; II : 409-30.

I I. Sai P Boitard C, Debray-Sachs M, Pouplard A, Assan R, Hamburger J. Complement fixing islet cell antibodies from some diabetic patients alter insulin release in vitro. Diabetes 1981 ; $30: 1051-7$

12. Sai $P$, Kremer $M$, Nomballais MF, Aillet $G$. Antibodies spontaneous by bound to islet cells in moins de 6 mois après le début clinique de l'affection.

L'infiltration lymphocytaire n'est présente que dans les îlots contenant des cellules bêta-langerhansiennes encore reconnaissables. Les lymphocytes disparaissent au contraire des îlots sans cellules bêta. Ils réapparaissent au contact des foyers de régénération des cellules bêta : ceci suggère que les lymphocytes sont attirés par un antigène porté par les cellules bêta, mais non par les cellules à glucagon, à somatostatine ou à PP [3].

On cherche actuellement à identifier les sous-populations de lymphocytes infiltrant les îlots, au moyen d'anticorps monoclonaux dirigés contre les marqueurs de surface correspondants. Jusqu'à présent il apparaît que les lymphocytes B (sécrétant les anticorps), mais aussi des lymphocytes $T$ appartenant à diverses sous-populations sont présents dans l'îlot.

Un autre effort porte sur la caractérisation des antigènes du complexe majeur d'histocompatibilité, en particulier de classe II (marqueurs HLA-DR), sur certaines cellules insulaires. Il semble que les cellules cibles (cellules bêta sécrétant l'insuline) expriment le HLA-DR, et pourraient présenter l'antigène aux lymphocytes tout comme le font, dans des conditions plus traditionnelles, les macrophages et les cellules dendritiques $[4,5]$. Cette constatation, si elle est confirmée, pourrait avoir d'importantes implications physiopathologiques. Elle pourrait être analogue à l'expression du HLA DR par les cellules cibles dans d'autres tissus qui sont l'objet d'une agression autoimmune : cellules thyroïdiennes dans la maladie de Basedow et certaines thyroïdites, cellules des canaux biliaires dans la cirrhose biliaire primitive $[4,5]$.

$\mathrm{La}$ présence de lésions insulaires inflammatoires multi-focales dans le pancréas de diabétique de type 1 soulève la question d'une physiopathologie virale. Une atteinte virale a été incriminée dans certaines observations cliniques privilégiées mais qui demeurent très rares [6]. Une atteinte insulaire inflammatoire et nécrotique a pu être reproduite expérimentalement dans certaines souches de souris par cer- tains virus coxsackie pancréatotropes. Cependant, très peu de virus présentent un effet cytolytique direct sur les îlots : c'est pourtant le cas du cytomégalovirus. Dans les observations anatomocliniques de diabète avec insulite survenu peu de jours après une affection virale à coxsackie, le caractère relativement ancien de l'insulite fait suspecter que le diabète a été en fait révélé et non causé par l'affection virale aiguë [7]. La recherche directe des antigènes viraux par immunofluorescence sur les coupes de pancréas de diabétiques récents est le plus souvent négative [7]. Certains virus paraissent capables de déclencher un processus autoimmun : le virus entraînant la sécrétion d'interféron gamma, lui-même entraînant l'expression du HLA DR par les cellules cibles. De fait, si cette hypothèse n'a jamais encore été vérifiée en clinique, elle a été reproduite expérimentalement : l'infestation de souris par un réovirus de type I induit chez ces souris un diabète sucré et une atteinte polyendocrinienne; les deux affections sont prévenues par l'administration d'un traitement immunosuppresseur [8].

\section{Arguments} immunologiques

Deux ordres d'observation s'alignent dans le domaine immunologique : d'une part, la caractérisation d'une immunité humorale et cellulaire dirigée contre les îlots par différents abords technologiques, témoins de la phase effectrice de l'immunité anti-îlot; et d'autre part la caractérisation plus délicate d'un déséquilibre dans la régulation de la reconnaissance du soi, avec un déséquilibre des sous-populations lymphocytaires T (tableau III).

Anticorps anti-îlot, immunité cellulaire spécifique et ADCC (immunité cellulaire dépendante d'anticorps) ont tous trois été caractérisés dans le diabète sucré de type 1 humain. Nous envisagerons essentiellement l'immunité humorale et l'immunité cellulaire.

Plusieurs techniques ont été utilisées pour caractériser les anticorps anti-îlot (tableau IV) :

Tout d'abord les techniques d'immunofluorescence à deux anti- 


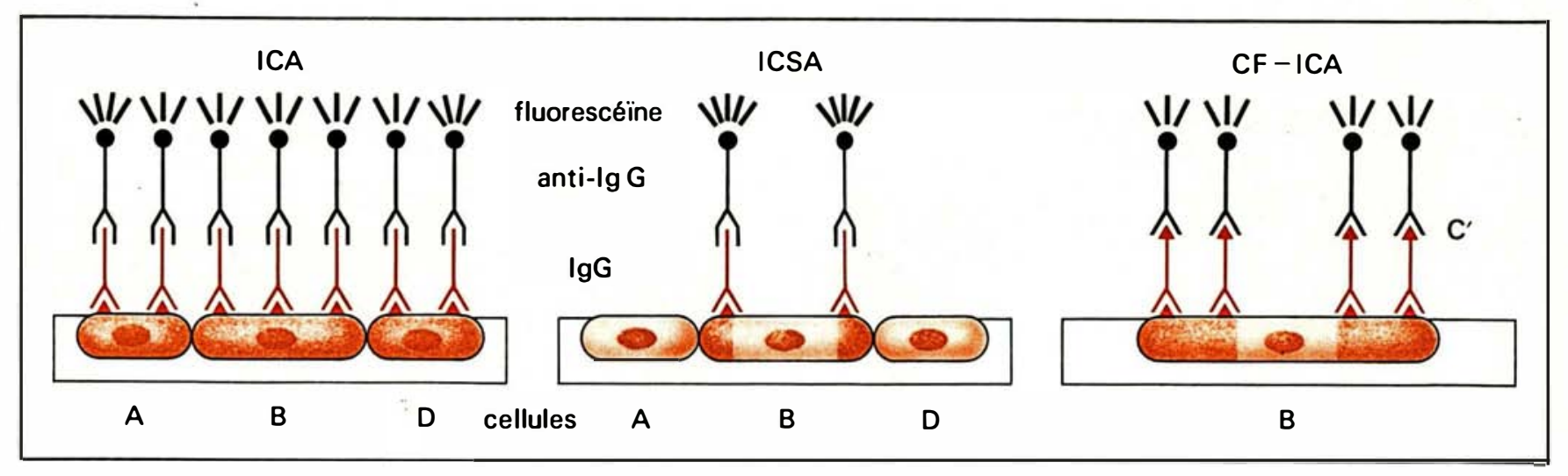

Figure 2. Représentation schématique de la recherche des anticorps anti-îlots en fluorescence.

ICA (islet cell antibodies) =anticorps dirigés contre les cellules des îlots de Langerhans.

ICSA (surface islet cell antibodies) =anticorps dirigés contre la membrane des cellules insulaires, spécifiques des cellules $\beta$ insulinosécrétrices.

$C F-I C A$ (complément fixing-ICA) = anticor ps fixant le complément et dirigés contre les cellules insulaires.

Les cellules $A, B, D$ figurées dans la coupe de pancréas correspondent aux cellules sécrétant glucagon, insuline, somatostatine. Les triangles noirs situés sur les membranes cellulaires symbolisent les épitopes de lantigène; les triangles marqués $C^{\prime}$ symbolisent le complément.

corps utilisant un substrat constitué de pancréas humain (pancréas adulte de groupe sanguin $\mathrm{ORh}$ négatif, pancréas foetal) coupé au cryostat, non fixé [9], qui permettent de caractériser ( $f i g .2)$ :

- des ICA (islet cell antibodies) se fixant sur le cytosol de toutes les cellules de l'îlot. Le second anticorps est un sérum anti-immunoglobuline (anti IgG) fluorescent.C'est la technique la plus sensible permettant l'évaluation du titre des anticorps sériques. C'est aussi la moins signicative du point de vue physiopathologique : il est difficile de concevoir que les anticorps se fixant sur tous les types de cellules insulaires lèsent électivement les cellules insulino-sécrétrices;

- des ICSA (islet cell surface antibodies) liant seulement la membrane des cellules insulaires et plus précisément des cellules insulinosécrétrices. Les ICSA sont de signification physiopathologique plus précise, étant des anticorps cytotoxiques et spécifiques des cellules bêta-langerhansiennes, mais ils sont de caractérisation plus délicates et donnent une fluorescence moins intense que les ICA;

- les CF-ICA (complement fixing-

$m / s n^{\circ} 4$ juin-juillet 85 islet cell antibodies) : après incubation du sérum à tester sur la coupe, du complément exogène est ajouté, et le second anticorps, lié à la fluorescéine, est un anticorps anticomplément. Ces anticorps évoquent fortement, comme les précédents, la présence d'anticorps cytotoxiques.

On utilise également des techniques de liaison de la protéine A staphylococcique marquée par ${ }^{125} \mathrm{I}$ : dans certains systèmes immunologiques (mais avec des variations selon les espèces) la protéïne $A$ iodée se fixe, tout comme le complément, sur les complexes antigènes-anticorps. La caractérisation se ramène à un comptage de radio-activité fixée aux cellules insulaires [10].

Une autre technique est l'inhibition fonctionnelle sélective de la sécrétion d'insuline dans ce système [11]. Des îlots normaux (humains, murins) sont isolés et incubés avec le sérum à tester, puis exposés à l'arginine. Cet acide aminé stimule normalement les sécrétions d'insuline, glucagon et somatostatine. Lorsqu'existe un anticorps anti-îlot spécifique des cellules bêta, l'inhibition de la seule sécrétion d'insuline et non des sécrétions de glucagon et
Tableau III

ANOMALIES IMMUNITAIRES DANS LE DIABËTE DE TYPE 1

- anticorps anti-îlots

- immunité cellulaire anti-îlots

- Iymphopénie T

- déséquilibre des sous-populations $T$

- déficits immunitaires secondaires au mauvais contrôle metabolique

\section{Tableau IV \\ PRINCIPE DES. MÉTHODES DE RECHERCHE \\ DES ANTICORPS ANTI-ILLOTS}

anticorps liant les cellules insulaires

- fluorescence (ICA-ICSA-CF-

ICA)

- protéine $A$ marquée par ${ }^{125}$

- ELISA

- détection d'IgG dans les îlots

anticorps cytotoxiques

- libération de ${ }^{51} \mathrm{Cr}$ ou ${ }^{3} \mathrm{H}$-Leu par les cellules insulaires marquées

- pénétration par les colorants post-vitaux (Ethidium Br; Rose Bengale)

- anticorps bloquant la sécrétion d'insuline in vitro 


\section{REFERENCES}

13. Irvine WJ, Mc Callum CJ, Gray RS, Duncan LJP. Pancreatic islet-cell antibodies mellitus correlated with the duration and type of diabetes, coexistent autoimmune disease, and HLA type. Diabetes 1 977; $26: 1$ 38-47.

14. Srikanta S, Ganda O, Eisenbarth G, Soeldner JS. Islet cell antibodies and beta-cell function in monozygotic triplets and twins initially discordant for type I diabetes mellitus. $N$ Engl 7 Med I 983;308:322-5

1 5. Nerup J, Lernmark A. Autoimmunity in diabetes mellitus. Am 7 Med 1981; 70; 1 35-41.

16. Boitard C, Debray-Sachs M, Pouplard A, Assan R, Hamburger J. Lymphocytes from diabetics suppress insulin release in vitro. Diabetologia $1981 ; 21: 41-6$.

17. Boitard C, Chatenoud LN, Debray-Sachs M In vitro inhibition of pancreatic $B$ cells by lymphocytes : a $\mathrm{T}$ cell phenomenon. 7 Immunol 1983; 1 $29: 2529-31$.

18. Arquilla ER, Stenger D. Effects of diabetes on immune function. In : Andreani D, Dimario U, Federlin KF, Heding LG, eds. Immunology of diabetes. London: Kimpton Medical Publications, $1984: 265-75$.

19. Lernmark A. Cell-mediated immunity in type I diabetes: update 84. In : Andreani D, Dimario U, Federlin KF, Heding LG, eds. Immunology of diabetes. London: Kimpton Medical Publications, $1984:$ I 2 I -3 I.

20. Pozzili P, Zuccarini O, Iavicoli $M$, et al. Monoclonal-antibodies defined abnormalities of $T$ lymphocytes in type $I$ (insulin-dependent) diabetes. Diabetes 1983, $32: 91-4$.

2 I. Dausset J, Hors J. Immunogenetics of insulin-dependent juveniles diabetes. Diabetes Research 1984; 1 : 1 i 5-23.

22. Cohen D, Cohen O, Marcadet A, et al. HLA class 2 beta-subunit restriction fragments differenciate among HLA $\mathrm{DR}_{2}$ individuals in insulindependent diabetes and multiple sclerosis. Proc Natl Acad Sci USA 1984; 81 : 1774-8.

23. Yale JF, Marliss EB. Altered immunity and diabetes in the BB rat. Clin Exp Immunol. 1984; $57: 1-11$.

24. Rabinowe SL, Eisenbarth GS. Immunotherapy of type I (insulin-dependent) diabetes mellitus. In : Andrani D, Dimario U, Federlin KF Heding LG, Eds. Immunology of diabetes. London : Kimpton Medical Publications, 1984 : I 7 I-5.

25. Stiller CR, Dupre J, Gent M, et al. Effects of cyclosporine immunosuppression in insulindependent diabetes mellitus of recent onset. Science 1 984; $223: 1362-7$.

26. Assan R., Feutren G, Laborie C, et al. Metabolic and immunological influences of cyclosporine. A treatment in recent onset type I (insulindependent) diabetics. Lancet $1985 ;$ i : 67-71.

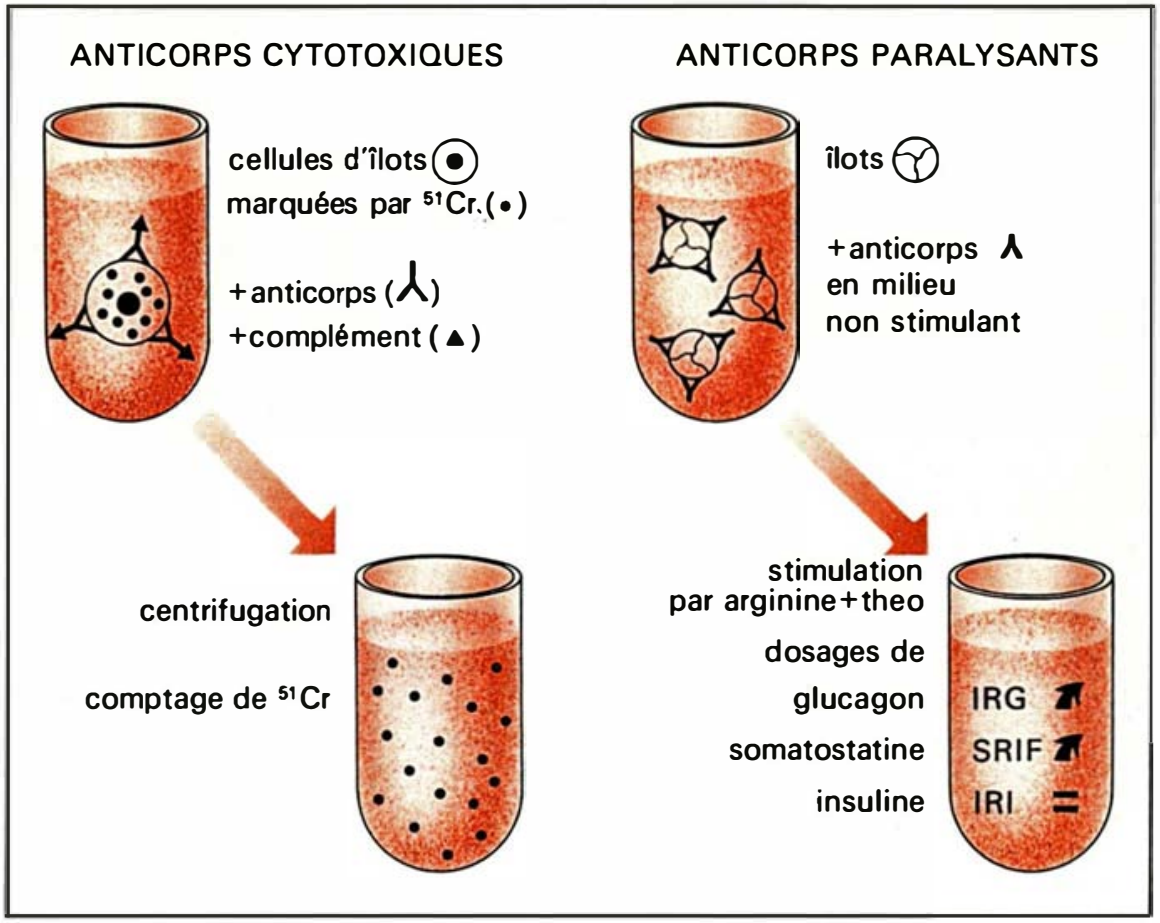

Figure 3. Représentation schématique de la recherche des anti-corps cytotoxiques pour les cellules insulaires (à gauche) et des anticorps bloquant l'insulinosécrétion (à droite).

${ }^{51} \mathrm{Cr}$ : chrome-5I; Théo : théophylline; IRI : insuline; IRG : glucagon. SRIF : somatostatine.

de somatostatine est très évocatrice d'une atteinte élective des cellules bêta-langerhansiennes (figure 3, panneau de droite).

Enfin, des variantes des techniques de microcytotoxicité utilisent la pénétration d'un colorant post-vital, bromure d'éthidium par exemple ou la libération d'un isotope $\left({ }^{51} \mathrm{Cr},{ }^{3} \mathrm{H}\right.$ Leu) incorporé aux cellules insulaires (figure 3, panneau de gauche). Des variantes des techniques de liaison utilisent le principe de la méthode immunoenzymatique (ELISA) : des cellules insulaires sont fixées aux parois du support (plaque de microtitration); l'anticorps anti-îlot se lie à ces cellules; une antiglobuline couplée à un substrat d'enzyme est ajoutée et révélée (en fluorimétrie ou colorimétrie) par l'enzyme appropriée.

\section{Analyse critique}

Les résultats de ces diverses techniques doivent être envisagés de façon critique à un double point de vue : méthodologique et physiopatholo- gique. Du point de vue méthodologique, les techniques de fluorescence (ICA) sont les mieux établies et constituent une sorte de référence.

Les résultats ont d'autant plus de valeur que les épitopes de la cible antigénique sont d'origine humaine, non remaniés par la fixation histologique. L'utilisation de substrats d'espèces différentes (primate, rongeur de laboratoire) nécessite en toute rigueur des adsorptions (ou épuisements) des sérums avec les poudres d'organes appropriés. L'utilisation comme substrats de cellulaires insulaires transformées en cultures (insulinomes de rats RIN 5F) est commode mais la résurgence $d$ 'antigènes tumoraux accroît les risques de discordance entre la cible in vitro et les îlots du malade lui-même. A l'intérieur même de l'îlot, la fixation sélective des anticorps sur les cellules bêtalangerhansiennes (ICSA) ou l'inhibition sélective de la sécrétion d'insuline in vitro apporte des garanties de spécificité et de signification 
physiopathologique accrue à l'anticorps ainsi caractérisé.

$\mathrm{Du}$ point de vue physiopathologique en effet, au sein d'une population polyclonale d'anticorps, certains peuvent n'être que des marqueurs secondaires à l'agression immunitaire alors que d'autres ou un seul d'entre-eux pourraient (ou pourrait) contribuer à la lésion des cellules insulinosécrétantes. Des dépôts d'immunoglobuline ont été récemment caractérisés au sein même des îlots de diabétiques humains de type 1 récemment diagnostiqués [12]. Les ICA sont présents dans le sérum de $80 \%$ environ des diabétiques de type 1 au début; leur titre et la fréquence de leur détection se réduit avec le temps. Les ICA ont été détectés chez des sujets apparentés aux diabétiques de type 1, précédant parfois l'apparition du diabète, ou disparaissant progressivement en l'absence d'une émergence clinique de la maladie [13]. Dans certaines circonstances privilégiées, telle que l'étude longitudinale de triplés homozygotes dont l'un était diabétique, la présence d'ICA a constitué une marque précoce, précédant l'altération de l'insulinosécrétion (abolition de la réponse insulinosécrétoire précoce au glucose intra-veineux) et l'apparition du diabète [14].

Cependant, en dehors de ces cas privilégiés, la présence d'ICA dans le sérum ne constitue pas actuellement un index prédictif fiable du diabète de type 1 .

Plus délicate encore est la mise en évidence de l'immunité cellulaire dirigée contre les îlots. A la classique technique d'inhibition, de migration des lymphocytes [15], s'ajoute aujourd'hui l'inhibition de la sécrétion d'insuline in vitro [16] : des cellules langerhansiennes normales sont incubées avec des lymphocytes à tester. La sécrétion d'insuline est électivement inhibée et ceci par les seuls lymphocytes des diabétiques de type 1 [16]. Ce sont les lymphocytes $T$ qui interviennent dans ce phénomène [17]. Lorsque les cellules insulaires cibles sont d'origine murine, il est clair que le principe de la restriction HLA rend délicate l'interprétation de ce thème. Celui-ci reste la meilleure évidence directe du rôle des lym- phocytes dans le déficit insulino sécrétoire des diabétiques de type 1. Parmi les anomalies de la régulation immunitaire chez le diabétique, il importe de distinguer les anomalies secondaires à la carence insulinique (c'est-à-dire au syndrome diabétique lui-même) des anomalies primitives. La carence insulinique induit une réduction de la sécrétion d'anticorps, de la phagocytose pour les bactéries, et de certaines manifestations de l'immunité cellulaire [18].

\section{Autres anomalies immunitaires}

Ces anomalies sont corrigées par la restauration d'une imprégnation insulinique appropriée.

Bien différentes sont les anomalies que l'on rencontre au début du diabète et qui paraissent indépendantes de la carence insulinique : augmentation du nombre de lymphocytes circulants activés augmentation du nombre des cellules NK (natural killer), et déséquilibre des sous-populations lymphocytaires [19]. Il a été décrit en effet une élévation précoce et transitoire du rapport lymphocytes helper sur lymphocytes supresseurs, et le développement d'une lymphopénie $\mathrm{T}$ progressive [20]. Ces anomalies ne sont pas sans rappeler celles observées dans divers syndromes de diabète spontané chez les rongeurs avec immunité anti-îlot comme on le verra plus loin.

\section{-Arguments génétiques}

Le diabète sucré de type 1 est électivement associé à certains marqueurs génétiques du complexe majeur d'histocompatibilité : HLA B8, B15, B18, DR3 et DR4 (figure 4). $\mathrm{Au}$ contraire, les marqueurs B7 et DR2 sont associés à un moindre risque de diabète sucré. D'autres marqueurs génétiques polymorphes situés non sur les membranes cellulaires, mais dans le plasma, sont associés au diabète sucré de type 1 avec une fréquence supérireure à celle rencontrée dans une population non sélectionnée : facteur BfF1 de la properdine, facteur B1Qo du complément, déficit en C3 du complément. L'intérêt se déplace depuis peu de ces produits (antigènes membranaires de classe I et II, antigènes sériques de classe I) aux gènes qui les codent. Le découpage de l'ADN chromosomique par les enzymes de restriction, suivi de la révélation des fragments d'ADN par les sondes radioactives appropriées suggèrent que la composition

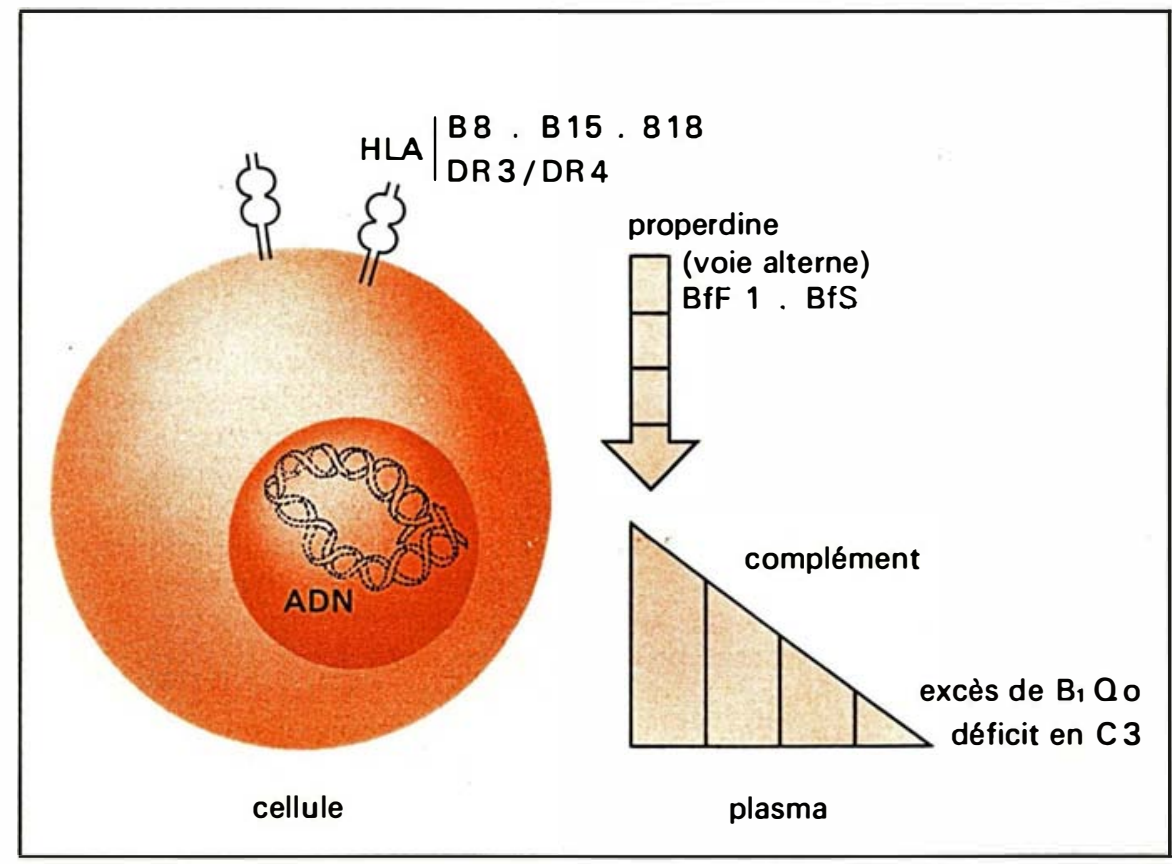

Figure 4. Marqueurs génétiques (sériques, membranaires, nucléaires) et diabéte sucré de type 1. 
Tableau V

HLA, ADN $(a+b+)$ ET DIABËTE DE TYPE 1

(fréquence en \%)

marqueurs

DR3
DR4
DR3/DR4
ADN $(a+b+)$ DR3/DR4
DR2
DR1

diabétiques témoins

$\begin{array}{rc}40 & 15 \\ 60 & 15 \\ 30 & 1 \\ 30 & 0,1 \\ 1 & 20 \\ 20 & 15\end{array}$

Les caractères $a^{+}$et $b^{+}$apparaissent après découpage de l'ADN, électrophorèse sur gel d'agarose et reconnaissance des fragments par les sondes radio-actives appropriées. D'après D. Cohen 1985.

même du gène DR3 est particulière chez les diabétiques de type 1 . Ainsi la grande fréquence reconnue des gènes DR3, DR4 et de l'haplotype DR3/DR4 chez les diabétiques de type 1 pourrait être associée à une anomalie qualitative du DR2 et du DR3 [21, 22].

Cette physionomie particulière, chez les diabétiques de type 1 , des gènes polymorphes situés sur le bras court du chromosome 6 soulève deux ordres d'intérêt. Le premier pratique : la détection précoce du diabète de type 1 et son identification nosologique sont en passe d'avoir des retombées thérapeutiques. Le rendement sociomédical de ce dépistage pourrait donc être accru par la caractérisation de l'ADN (tableau V). Le second intérêt est fondamental : la proximité sur le chromosome 6 des gènes de régulation de la réponse immune et des gènes d'histocompatibilité polymorphe pourrait rendre compte d'anomalies de la reconnaissance du soi chez les diabétiques de type 1 .

\section{Modèles animaux}

On connaît depuis peu d'années des modèles de diabète insulinoprive spontané chez les animaux, associé à une autoimmunité anti-îlot. Il faut y ajouter des modèles expérimentaux : le diabète induit par de faibles doses de streptozotocine et la polyendocrinopathie induite par les réovirus chez la souris.

Le diabète du rat $\mathrm{BB}$ est particu- lièrement intéressant par ses analogies avec le diabète de type 1 humain. Ce mutant de rat Wistar, découvert il y a quelques années dans un élevage canadien (Bio Breeding) développe un diabète cétosique insulinoprive atteignant 40 à $60 \%$ des animaux des deux sexes dans les portées à haut risque. Ce diabète apparaît vers le $90^{\circ}$ jour de la vie, et il est précédé de 8 à 15 jours par une insuline, très semblable à une insulite humaine. Une thyroïdite autoimmune apparaît plus tardivement. Une lymphopénie $\mathrm{T}$ précoce, un déséquilibre des sous-populations lymphocytaires $\mathrm{T}$ sont associés à une autoimmunité anti-îlot, humorale et cellulaire.

Insulite et diabète sont transmissibles par transfert de lymphocytes à des animaux syngéniques. Le diabète peut être prévenu par thymectomie néonatale, ou par transfusion de lymphocytes normaux, ou par greffe de moëlle néonatale, ou enfin par traitement immunosuppresseur (irradiation $\mathrm{X}$, sérum anti-lymphocyte, cyclosporine A). Tous ces traits soulignent le rôle déterminant des lymphocytes en particulier des lymphocytes $T$ dans la physiopathologie du syndrome.

Le diabète est associé à l'antigène RT1 du complexe majeur d'histocompatibilité. D'autres manifestations d'incompétence immunitaire sont présentes (anomalies de la réponse à la phytohémagglutinine, à la candidine etc.), de même que l'apparition relativement fréquente de lymphomes. Ce modèle intensivement étudié actuellement, s'est avéré particulièrement fructueux pour l'étude expérimentale du traitement immunosuppresseur. L'administration de cyclosporine A pendant 10 jours seulement avant la période d'apparition de l'insulite, prévient le développement de celleci ainsi que l'évolution vers le diabète [23].

\section{immunosuppresseur}

L'élaboration du concept d'immunité anti-îlot dans le diabète de type 1 conduisait logiquement depuis près de 5 ans, à des tentatives thérapeutiques immunosuppressives. Irradiation corporelle massive, traitement par le cyclophosphamide, plasmaphérèses, corticothérapie ont fait l'objet de tentatives décevantes [24]. L'utilisation plus récente de la cyclosporine $\mathrm{A}$ donne des résultats plus encourageants. Ceux-ci paraissent particulièrement intéressants en raison de leur signification physiopathologique et du problème de détection précoce du diabète qu'ils soulèvent.

\section{Résultats des essais pilotes}

La cyclosporine est un métabolite fongique qui inhibe électivement la fonction des lymphocytes $\mathrm{T}$ helper, la libération d'interleukine 2 et l'immunité cellulaire. Elle n'interfere ni avec l'hématopoïèse ni avec la mémoire immunitaire, ni pour l'essentiel avec l'immunité humorale. Elle a été utilisée à ce jour chez environ 19000 transplantés et chez près de 1000 sujets atteints d'affections autoimmunes. Pour ce qui est des diabétiques de type 1 , deux essais pilotes non randomisés ont été réalisés par Stiller et al. [25] sur une soixantaine de diabétiques récents et à Paris [26] sur 12 diabétiques récents. Dans les 2 cas, 30 à $40 \%$ des sujets ont pu interrompre totalement l'insulinothérapie en gardant des glycémies normales, cependant que la sécrétion d'insuline endogène réapparaissait. Une rémission incomplète de l'insulinodépendance était notée dans un autre tiers des sujets, les doses d'in- 
suline nécessaires au maintien de glycémies normales étant réduites de plus de $50 \%$. Une réduction marquée du rapport helper/suppresseurs et de l'immunité cellulaire anti-îlot était présente chez tous les sujets étudiés à Paris. L'interruption abrupte du traitement par la cyclosporine a été suivie d'une réascension des besoins en insuline. Au contraire, la réduction très progressive des doses de cyclosporine jusqu'à l'arrêt n'a pas été suivie à ce jour de rechute de l'insulinodépendance. Ces résultats encourageants ont conduit à l'élaboration de plusieurs essais multicentriques randomisés en France, en Europe, au Canada, et aux U.S.A. Ces essais sont en cours actuellement et portent au total sur plusieurs centaines de diabétiques récents.

Dans l'analyse des résultats des essais pilotes, la réduction des besoins en insuline exogène était rapide (quelques jours) et corrélée dans le temps avec l'institution du traitement immunosuppresseur. La réapparition rapide d'une sécrétion $d$ 'insuline endogène suggère qu'une régénération des cellules sécrétant l'insuline (ou une levée d'inhibition) est possible après l'éclosion clinique du diabète lorsque l'agression auto immunitaire est bloquée par la cyclosporine. Les rémissions ont été d'autant plus fréquentes que le diabète était plus récent. $\mathrm{Si}$ cette donnée recueillie sur un petit groupe se confirme, elle a d'importantes implications. L'utilisation d'un traitement immunosuppresseur devrait être aussi précoce que possible chez les diabétiques de type 1. Ceci conduit à rechercher une caractérisation aussi solide que possible des "pré-diabétiques" de type 1 . Chez le rat $\mathrm{BB}$, comme on la vu, l'efficacité de la cyclosporine pour prévenir le diabète est maximale lorsque le traitement est entrepris avant l'apparition de l'insulite. L'évaluation définitive de la valeur thérapeutique de cette tentative nécessite le recul du temps et l'analyse des résultats des essais randomisés. Dès à présent cependant, les résultats des essais pilotes constituent un argument physiopathologique important en faveur de l'origine autoimmune et du rôle des lymphocytes $\mathrm{T}$ dans l'apparition du diabète de type insulinodépendant.

L'initiation du processus autoimmunitaire reste un phénomène de déterminisme obscur. Pourquoi le sujet pourvu d'un capital génétique à haut risque déclenche-t-il insulite et diabète à un moment donné de sa vie? L'agression autoimmune de l'îlot est-elle en rapport avec une modification acquise d'un antigène insulaire (par un virus, par un agent toxique etc.) ou bien en rapport avec une propension innée du système immunitaire à la mauvaise reconnaissance du soi ?

\section{Les questions en suspens}

Le déclenchement du processus immunitaire nécessite la présentation de l'antigène aux lymphocytes $T$ par un macrophage, ou une cellule dentritique ainsi que l'expression sur la membrane de cette cellule de l'antigène de classe 2 HLA $\mathrm{DR}[4,5]$. Des observations privilégiées suggèrent que la cellule bêtalangerhansienne est capable d'exprimer le DR et de présenter elle-même l'auto-antigène. Des observations semblables ont été faites à propos d'autres affections autoimmunes, thyroïdite, hépatite chronique active [4]. Mais ces observations concernant la cellule d'îlot nécessitent une confirmation sur un plus grand nombre de cas. La caractérisation de l'antigène responsable de la réaction autoimmune est un autre point qui reste ouvert. Des antigènes relativement purifiés ont été obtenus à partir de membranes de cellules d'îlots, et se montrent capables de précipiter avec certains anticorps anti-îlots. Il n'est pas démontré cependant qu'il s'agisse de l'antigène responsable du déclenchement de la réaction autoimmune.

Parmi les nombreux points qui restent en suspens, un autre est celui du traitement immunosuppresseur; en effet s'il est efficace, on peut difficilement concevoir de maintenir indéfiniment des diabétiques sous traitement immunosuppresseurpar la cyclosporine $A$.

La recherche d'un traitement immunosuppresseur électif et potentiellement moins toxique doit donc être envisagée

\section{Summary}

Type 1 diabetes (insulindependent, without macroscopic pancreatic disease) is associated with inflammatory insulitis and with a humoral and cellular immunological reaction against islets, and, more precisely, against insulinsecreting cells. The value of immunological methods demonstrating this auto-immune reaction is critically evaluated in this article. Genetic markers (in serum, membranes and nuclei) are preferentially associated with type 1 diabetes. The recent description of animal models with immunological reactions directed against islets, and the obtention of remissions with immunosuppressive therapy in patients, support the concept of type 1 diabetes being an autoimmune disease. The nature of the antigen and its mode of presentation to helper $T$ lymphocytes remain unknown. Such a concept could have important clinical implications in the early detection of type 1 diabetes and pre-diabetic states, and in the optimisation of immunosuppressive treatment.

\section{Remerciements}

Nous remercions, pour leurs conseils et pour leurs discussions critiques et enrichissantes. messieurs les Professeurs J. Hamburger, J. F. Bach, J. Dausset, ainsi que les Docteurs C. Boitard, G. Feutren et D. Cohen.

\section{TIRES A PART \\ R. Assan : Hôpital Bichat, 18, rue Henri- Huchard, 75018 Paris.}

